PROCEEDINGS OF THE

AMERICAN MATHEMATICAL SOCIETY

Volume 136, Number 10, October 2008, Pages 3477-3482

S 0002-9939(08)09514-2

Article electronically published on May 23, 2008

\title{
A SUFFICIENT CONDITION FOR FINITE TIME BLOW UP OF THE NONLINEAR KLEIN-GORDON EQUATIONS WITH ARBITRARILY POSITIVE INITIAL ENERGY
}

\author{
YANJIN WANG \\ (Communicated by Ronald A. Fintushel)
}

\begin{abstract}
In this paper we consider the nonexistence of global solutions of a Klein-Gordon equation of the form

$$
u_{t t}-\Delta u+m^{2} u=f(u), \quad(t, x) \in[0, T) \times \mathbb{R}^{n} .
$$

Here $m \neq 0$ and the nonlinear power $f(u)$ satisfies some assumptions which will be stated later. We give a sufficient condition on the initial datum with arbitrarily high initial energy such that the solution of the above Klein-Gordon equation blows up in finite time.
\end{abstract}

\section{INTRODUCTION}

This paper studies the nonexistence of global solutions of the nonlinear KleinGordon equation

$$
\left\{\begin{aligned}
u_{t t}-\Delta u+m^{2} u=f(u), & (t, x) \in[0, T) \times \mathbb{R}^{n}, \\
u(0, x)=u_{0}(x), & x \in \mathbb{R}^{n}, \\
u_{t}(0, x)=u_{1}(x), & x \in \mathbb{R}^{n},
\end{aligned}\right.
$$

where $\Delta$ is a Laplacian operator on $\mathbb{R}^{n}(n \geq 1), u_{0}(x)$ and $u_{1}(x)$ are real valued functions, $m \neq 0$ is a real constant, and the right-hand side $f(u)$ is a real valued, nonlinear function of $u$. Without loss of generality, we may assume that $m=1$ throughout the paper.

The above problem has various applications in the area of nonlinear optics, plasma physics, fluid mechanics, etc. There is literature on the Cauchy problem for equation (1.1) (see, for instance, 3], 5], 6, 17, 13, [14, [15, [16] and the papers cited therein). The results about the blow up properties for the local solution of equation (1.1) are investigated by [1, 8], 12, 15], 16. For the typical form of the nonlinear power, $f(u)=|u|^{p-1} u$ with $1<p<\frac{n+2}{n-2}(n \geq 2)$, we refer to the reader the result [16, which established a sharp condition for the global existence and blow up of the solution of the Klein-Gordon equation (1.1). But in 16, the

Received by the editors November 10, 2006, and, in revised form, November 23, 2006.

2000 Mathematics Subject Classification. Primary 35L05, 35L15.

Key words and phrases. Klein-Gordon equation, blow up, positive initial energy.

This work was supported by a Japanese government scholarship. The author wishes to express his deep gratitude to Professor Hitoshi Kitada for his constant encouragement and kind guidance. Thanks also go to the referees for their comments and careful reading of the manuscript.

(C)2008 American Mathematical Society Reverts to public domain 28 years from publication 
initial energy had an upper bound. This motivates us to consider the problem of how to obtain the solution when the initial energy is arbitrarily large.

In this paper we investigate the above problem for equation (1.1) with the nonlinear power $f(u)$ which satisfies that there exists $\epsilon>0$ such that for any $s \in \mathbb{R}$,

$$
f(s) s \geq(2+\epsilon) F(s)
$$

where $F(s)=\int_{0}^{s} f(\xi) d \xi$.

For the above nonlinear power, the nonexistence of the global solution of an abstract wave equation (including the Klein-Gordon equation) was established in [8] when the initial energy was negative. However, until recently there has been very little work on the global existence and global nonexistence of the solutions of the initial value problem for Klein-Gordon equations when the initial energy is arbitrarily high.

The purpose of this paper is to establish a sufficient condition of the initial datum with arbitrarily high initial energy such that the corresponding local solution of the nonlinear Klein-Gordon equation (1.1) blows up in a finite time. As far as we know, this is the first blow up result for the Klein-Gordon equations with arbitrarily high initial energy on the whole space $\mathbb{R}^{n}$. Our proof is based on the concavity method which was first introduced by Levine $[\underline{8}, 9]$. We note that our proof is very simple and that it can be applied to some other equations.

Here we also refer the result of [10] to the reader. Levine and Todorova 10] studied a damped wave equation in the form

$$
\left\{\begin{array}{l}
u_{t t}+a\left|u_{t}\right|^{\rho-1} u_{t}-\Delta u=b|u|^{p-1} u-q(x)^{2} u \text { in }[0, T) \times \mathbb{R}^{n} \\
u(0, x)=u_{0}(x) \text { in } \mathbb{R}^{n} \\
u_{t}(0, x)=u_{1}(x) \text { in } \mathbb{R}^{n}
\end{array}\right.
$$

where $a, b>0, \rho \geq 1$, and $q(x)^{2} \geq 0$. They proved the existence of the initial datum with arbitrarily high initial energy such that the solution blows up in a finite time when $1 \leq \rho<p$. More recently, for the case, $\rho=1$ and $q(x)=0$, when the initial energy is arbitrarily high, Gazzola and Squassina [4 have obtained the blow up result on an open bounded Lipschitz subset of $\mathbb{R}^{n}$. We note that their proof cannot be extended to the whole space $\mathbb{R}^{n}$. Our proof can be easily adapted to the damped wave equation (1.3) with $\rho=1$ and $q(x)=m \neq 0$. In this paper we will not discuss it in detail but will make some remarks.

The paper is organized as follows. Section 2 introduces some notation and known results, and also states our main result. In Section 3 we prove the main result based on a concavity argument. We make some remarks in the last section.

\section{Preliminary And main Result}

Before we state the main result, we introduce some notation. We denote by $\|\cdot\|_{q}$ the $L^{q}\left(\mathbb{R}^{n}\right)$ norm for $1 \leq q \leq \infty$, and we define the spaces $H^{1}\left(\mathbb{R}^{n}\right)=\{u \in$ $\left.L^{2}\left(\mathbb{R}^{n}\right) ;\|u\|_{H^{1}\left(\mathbb{R}^{n}\right)}=\left\|(1-\Delta)^{1 / 2} u\right\|<\infty\right\}$ and $H_{0}^{1}\left(\mathbb{R}^{n}\right)=\left\{u \in H^{1}\left(\mathbb{R}^{n}\right) ; \operatorname{supp}(u)\right.$ is compact in $\left.\mathbb{R}^{n}\right\}$. For simplicity we will denote $\int_{\mathbb{R}^{n}}$ by $\int$. The notation $t \rightarrow T^{-}$ means $t<T$ and $t \rightarrow T$.

We first state the result of the local existence established in [3]. 
Theorem 2.1. Let the initial data $\left(u_{0}, u_{1}\right) \in H_{0}^{1}\left(\mathbb{R}^{n}\right) \times L^{2}\left(\mathbb{R}^{n}\right)$, and let $f$ satisfy the following conditions: $f(0)=0$,

$$
\left|f\left(\lambda_{1}\right)-f\left(\lambda_{2}\right)\right| \leq c\left(\left|\lambda_{1}\right|^{p-1}+\left|\lambda_{2}\right|^{p-1}\right)\left|\lambda_{1}-\lambda_{2}\right|
$$

for all $\lambda_{1}, \lambda_{2} \in \mathbb{R}$ and some constant $c>0$, and

$$
1<p<\frac{n}{n-2} \text { if } n \geq 3 ; \quad 1<p<\infty \text { if } n=1,2
$$

Then there is a unique local solution $u(t, x)$ of equation (1.1) on a maximal time interval $\left[0, T_{\max }\right)$ satisfying $u(0, x)=u_{0}(x)$ and $u_{t}(0, x)=u_{1}(x)$. In addition, $u(t, x)$ satisfies

$$
E(t)=E(0)
$$

where

$$
E(t)=\frac{1}{2} \int\left(\left|u_{t}(t, x)\right|^{2}+|u(t, x)|^{2}+|\nabla u(t, x)|^{2}-2 F(u(t, x))\right) d x .
$$

In order to state the main result, we next define a function,

$$
I(u)=\int\left(|u(x)|^{2}+|\nabla u(x)|^{2}\right) d x-\int f(u(x)) u(x) d x .
$$

Now we are in the position to state our main result:

Theorem 2.2. Let $f(s)$ satisfy the assumption (1.2). If the initial datum $\left(u_{0}, u_{1}\right) \in$ $H_{0}^{1}\left(\mathbb{R}^{n}\right) \times L^{2}\left(\mathbb{R}^{n}\right)$ satisfies that

$$
\begin{aligned}
& E(0)>0 \\
& \left\|u_{0}\right\|^{2} \geq \frac{2(2+\epsilon)}{\epsilon} E(0), \\
& I\left(u_{0}\right)<0 \\
& \int u_{0} u_{1} d x>0
\end{aligned}
$$

where $\epsilon>0$ is the constant in (1.2), then the corresponding local solution $u(t, x)$ of equation (1.1) will blow up in a finite time $T_{\max }<\infty$; that is to say,

$$
\lim _{t \rightarrow T_{\max }^{-}}\|u(t, \cdot)\|^{2} \rightarrow \infty
$$

\section{Proof of Theorem 2.2}

The proof is split into two steps.

We first prove that

$$
\begin{aligned}
& \|u(t, \cdot)\|^{2} \geq \frac{2(2+\epsilon)}{\epsilon} E(0), \\
& I(u(t, \cdot))<0
\end{aligned}
$$

for every $t \in\left[0, T_{\max }\right)$.

We prove $I(u(t, \cdot))<0$ for every $t \in\left[0, T_{\max }\right)$. Suppose to the contrary that there exists a time $T>0$ such that

$$
T=\min \left\{t \in\left(0, T_{\max }\right) ; I(u(t, \cdot))=0\right\} .
$$

We now define an auxiliary function:

$$
G(t)=\int|u(t, x)|^{2} d x
$$


By simple computations we have

$$
\begin{aligned}
& G^{\prime}(t)=2 \int u u_{t} d x \\
& \frac{1}{2} G^{\prime \prime}(t)=\int\left|u_{t}\right|^{2} d x+\int\left(f(u) u-|\nabla u|^{2}-|u|^{2}\right) d x .
\end{aligned}
$$

Noting the assumption $I(u(t, \cdot))<0$ for every $t \in[0, T)$, we obtain $G^{\prime \prime}(t)>0$ for every $t \in[0, T)$, which implies that $G^{\prime}(t)$ is strictly increasing on $[0, T)$. Then by (2.9) we see that $G^{\prime}(t)>0$ for every $t \in[0, T)$. In other words, we obtain that $G(t)$ is also strictly increasing on $[0, T)$. Thus we obtain

$$
G(t)>G(0) \geq \frac{2(2+\epsilon)}{\epsilon} E(0)
$$

for every $t \in(0, T)$. From the continuity of $u(t, x)$ at $t=T$ it follows that

$$
G(T)=\|u(T, \cdot)\|^{2}>\frac{2(2+\epsilon)}{\epsilon} E(0) .
$$

But, by (2.4) it is obvious that

$$
\|u(T, \cdot)\|^{2}+\|\nabla u(T, \cdot)\|^{2}-2 \int F(u(T, x)) d x \leq 2 E(T)=2 E(0) .
$$

Noting the assumption $I(u(T, \cdot))=0$ and (1.2), we have

$$
\|u(T, \cdot)\|^{2}+\|\nabla u(T, \cdot)\|^{2} \geq(2+\epsilon) \int F(u(T, x)) d x .
$$

We now obtain

$$
\|u(T, \cdot)\|^{2}+\|\nabla u(T, \cdot)\|^{2} \leq \frac{2(2+\epsilon)}{\epsilon} E(0) .
$$

Obviously there is a contradiction between (3.6) and (3.7). Thus we have proved that for every $t \in\left[0, T_{\max }\right)$

$$
I(u(t, \cdot))<0 .
$$

By the proof above, we also see that if $I(u(t, \cdot))<0$ for every $t \in\left[0, T_{\max }\right)$, then $G(t)$ is strictly increasing on $\left[0, T_{\max }\right)$. Thus (3.8) implies

$$
\|u(t, \cdot)\|^{2}>\frac{2(2+\epsilon)}{\epsilon} E(0)
$$

for every $t \in\left(0, T_{\max }\right)$.

Therefore we have completed the proof of (3.1) and (3.2). We next prove the blow up result of equation (1.1).

By (3.5) and (3.9), we see that

$$
\begin{aligned}
\frac{1}{2} G^{\prime \prime}(t) & =\int\left|u_{t}\right|^{2} d x-\int\left(\left|u^{2}\right|+|\nabla u|^{2}\right) d x+\int f(u) u d x \\
& \geq\left(2+\frac{\epsilon}{2}\right) \int\left|u_{t}\right|^{2} d x+\frac{\epsilon}{2} \int\left(|u|^{2}+|\nabla u|^{2}\right) d x-(2+\epsilon) E(0) \\
& \geq\left(2+\frac{\epsilon}{2}\right) \int\left|u_{t}\right|^{2} d x
\end{aligned}
$$


By the Schwarz inequality, we therefore have

$$
\begin{aligned}
G^{\prime \prime}(t) G(t)-\frac{4+\epsilon}{4}\left(G^{\prime}(t)\right)^{2} & \geq(4+\epsilon)\left\{\int|u|^{2} d x \int\left|u_{t}\right|^{2} d x-\left(\int u u_{t} d x\right)^{2}\right\} \\
& \geq 0
\end{aligned}
$$

for every $t \in\left[0, T_{\max }\right)$. As $\frac{4+\epsilon}{4}>1$, letting $\alpha=\frac{\epsilon}{4}$, we have

$$
\begin{aligned}
\left(G^{-\alpha}\right)^{\prime} & =-\alpha G^{-\alpha-1} G^{\prime}(t)<0, \\
\left(G^{-\alpha}\right)^{\prime \prime} & =-\alpha G^{-\alpha-2}\left[G^{\prime \prime}(t) G(t)-\frac{4+\epsilon}{4}\left(G^{\prime}(t)\right)^{2}\right] \\
& \leq 0
\end{aligned}
$$

for every $t \in\left(0, T_{\max }\right)$, which means that the function $G^{-\alpha}$ is concave. Obviously $G(0)>0$; then from (3.12) it follows that the function $G^{-\alpha} \rightarrow 0$ when $t<T_{\max }$ and $t \rightarrow T_{\max }\left(T_{\max }<\frac{\epsilon G(0)}{4 G^{\prime}(0)}\right)$. Therefore, we see that there exists a finite time $T_{\max }>0$ such that

$$
\lim _{t \rightarrow T_{\max }^{-}}\|u(t, \cdot)\|^{2} \rightarrow \infty .
$$

Thus, the proof of Theorem 2.2 is completed.

\section{SOME REMARKS}

First we consider a special case of the nonlinear power $f(u)$, which is the typical form of the nonlinear power $f(u)=|u|^{p-1} u$ with $1<p<\frac{n+2}{n-2}$ and $n \geq 2$. We define the following action:

$$
J(u)=\frac{1}{2} \int\left(|u|^{2}+|\nabla u|^{2}-\frac{2}{p+1}|u|^{p+1}\right) d x .
$$

As in [2, we have that

Theorem 4.1. There exists $\bar{u}(x) \in M$ such that

$$
d=\inf _{u \in M} J(u)=J(\bar{u}),
$$

where $M=\left\{u \in H^{1}\left(\mathbb{R}^{n}\right) ; I(u)=0, u \neq 0\right\}$. Moreover, $\bar{u}$ is the ground state of the elliptic equation

$$
-\Delta u+u=u^{p}, \quad u \in H^{1}\left(\mathbb{R}^{n}\right) .
$$

Thus, when $E(0)<d$, as in the first part of the proof of Theorem 2.2, we can prove that if there exists $T$ such that $I(u(T, \cdot))=0$, the function $u(T, x) \in$ $H_{0}^{1}\left(\mathbb{R}^{n}\right) \backslash\{0\}$ satisfies

$$
\begin{aligned}
& I(u(T, \cdot))=0, \\
& J(u(T, \cdot)) \leq E(T)=E(0)<d .
\end{aligned}
$$

This contradicts Theorem 4.1. Thus for the case $E(0)<d$ the blow up result holds. This reproduces the results in [16] and [11]. 
Secondly, we consider an example to which our method is applicable. For the damped wave equation (1.3) with $\rho=1$ and $q(x)^{2}=m^{2}=1$, we define the auxiliary function $G(t)$ corresponding to (3.3) in the form

$$
G(t)=\int|u(t, x)|^{2} d x+\int_{0}^{t}\|u(\tau, \cdot)\|^{2} d \tau+\left(T_{0}-t\right)\left\|u_{0}\right\|^{2},
$$

where $T_{0}>0$ is some constant. Then the argument in [4] proves $I(u(t))<0$. Thus similarly to the proof of our Theorem 2.2 , we get the blow up result for the damped wave equation.

\section{REFERENCES}

1. J.M. Ball, Finite time blow-up in nonlinear problems, in: M.G. Grandall (Ed.), Nonlinear Evolution Equations, Academic Press, New York, 1978, 189-205. MR513819 (80c:35012)

2. H. Berestycki and T. Cazenave, Instabilité des états stationnaires dans les équations de Schrödinger et de Klein-Gordon non linéaires, C. R. Acad. Sci. Paris 293(1981) 489-492. MR646873 (84f:35120)

3. T. Cazenave, Uniform estimates for solutions of nonlinear Klein-Gordon equations, Journal of Functional Analysis 60(1985) 36-55. MR780103 (86f:35157)

4. F. Gazzola and M. Squassina, Global solutions and finite time blow up for damped semilinear wave equations, Ann. Inst. H. Poincaré Anal. Non Linéaire 23(2006) 185-207. MR 2201151 (2007c:35118)

5. J. Ginibre and G. Velo, The global Cauchy problem for the nonlinear Klein-Gordon equation, Math. Z. 189(1985) 487-505. MR786279 (86f:35149)

6. J. Ginibre and G. Velo, The global Cauchy problem for the nonlinear Klein-Gordon equation. II, Ann. Inst. H. Poincaré Anal. Non Linéaire 6(1989) 15-35. MR984146 (90b:35153)

7. S. Klainerman, Global existence of small amplitude solutions to nonlinear Klein-Gordon equations in four space-time dimensions, Commun. Pure Appl. Math. 38(1985) 631-641. MR $803252(87 \mathrm{e}: 35080)$

8. H.A. Levine, Instability and nonexistence of global solutions of nonlinear wave equations of the form $P u_{t t}=-A u+\mathcal{F}(u)$, Trans. Amer. Math. Soc. 192(1974) 1-21. MR 0344697(49:9436)

9. H.A. Levine, Some additional remarks on the nonexistence of global solutions to nonlinear wave equations, SIAM J. Math. Anal. 5(1974) 138-146. MR0399682 (53:3525)

10. H.A. Levine and G. Todorova, Blow up of solutions of the Cauchy problem for a wave equation with nonlinear damping and source terms and positive initial energy, Proc. Amer. Math. Soc. 129(2001) 793-805. MR 1792187 (2001k:35212)

11. Y. Liu, M. Ohta and G. Todorova, Strong instability of solitary waves for nonlinear KleinGordon equations and generalized Boussinesq equations, Ann. Inst. H. Poincaré Anal. Non Linéaire 24(2007) 539-548. MR2334991

12. L.E. Payne and D.H. Sattinger, Saddle points and instability of nonlinear hyperbolic equations, Israel J. Math. 22(1975) 273-303. MR0402291 (53:6112)

13. H. Pecher, $L^{p}-$ Abschätzungen und klassische Lösungen für nichtlineare Wellengleichungen. I, Math. Z. 150(1976) 159-183. MR0435604(55:8563a)

14. J. Simon and E. Taflin, The Cauchy problem for nonlinear Klein-Gordon equations, Commun. Math. Phys. 152(1993) 433-478. MR:1213298 (94d:35110)

15. W. Strauss, Nonlinear wave equations, CBMS Regional Conference Series in Math., 73, Amer. Math. Soc., Providence, RI, 1989. MR1032250 (91g:35002)

16. J. Zhang, Sharp conditions of global existence for nonlinear Schrödinger and Klein-Gordon equations, Nonlinear Analysis 48(2002) 191-207. MR.1870752 (2002h:35303)

Graduate School of Mathematics, University of Tokyo, 3-8-1 Komaba, Meguro, TOKYO, 153-8914, JAPAN Current address: Institute of Applied Physics and Computational Mathematics, P.O. Box 8009-15, Beijing, 100088, People's Republic of China

E-mail address: wangyj@ms.u-tokyo.ac.jp

E-mail address: wang-jasonyj2002@yahoo.com 